\title{
TRACES AND RESIDUES
}

\author{
AMNON NEEMAN
}

\begin{abstract}
Let $f: X \longrightarrow Y$ be a separated morphism of noetherian schemes, and let $W \subset X$ be a union of closed subsets such that the restriction of $f$ to each of them is proper. In duality theory one considers trace maps $\mathbf{R} f_{*} \mathbf{R} \Gamma_{W} f^{!} \mathcal{O}_{Y} \longrightarrow \mathcal{O}_{Y}$. In a recent paper we gave a new construction of such a trace map, using a certain natural transformation $\psi(f): f^{\times} \longrightarrow f^{!}$. In this note we show how to compute it.

In duality theory there are abstract, functorial definitions and there are computationally useful formulas, but they are rarely the same. This makes the new approach remarkable.
\end{abstract}

\section{Contents}

0. Introduction 1

1. The map $\gamma_{f}$ is an isomorphism 3

2. Residues 8

References 11

\section{INTRODUCTION}

Let $\mathbb{S}_{\mathbf{e}}$ be the category whose objects are noetherian schemes, and the morphisms are the maps essentially of finite type. Let $f: X \longrightarrow Y$ be a morphism in $\mathbb{S}_{\mathbf{e}}$; there is a pushforward functor $\mathbf{R} f_{*}: \mathbf{D}_{\mathbf{q c}}(X) \longrightarrow \mathbf{D}_{\mathbf{q c}}(Y)$ which has a left adjoint $\mathbf{L} f^{*}$ and a right adjoint $f^{\times}$. A recent article [9] shows that there is also a fourth functor $f^{!}: \mathbf{D}_{\mathbf{q c}}(Y) \longrightarrow$ $\mathbf{D}_{\mathbf{q c}}(X)$, until recently it was only known to be well defined on $\mathbf{D}_{\mathbf{q c}}^{+}(Y) \subset \mathbf{D}_{\mathbf{q c}}(Y)$. The way $f^{!}$is traditionally defined is

(i) Factor $f: X \longrightarrow Y$ as $X \stackrel{g}{\longrightarrow} Z \stackrel{h}{\longrightarrow} Y$, with $g$ a localizing immersion and $h$ proper.

(ii) Set $f^{!}=g^{*} h^{\times}$.

With this definition it isn't clear that $f^{!}$is independent of the choice of factorization, for the unbounded derived category it wasn't known until the very recent [9]. Another recent article [7] produces a natural transformation $\psi(f): f^{\times} \longrightarrow f^{!}$and then uses it to define a trace map. One definition of the map $\psi(f)$ is as follows:

2000 Mathematics Subject Classification. Primary 14F05.

Key words and phrases. Derived categories, Grothendieck duality.

The research was partly supported by the Australian Research Council. 
(iii) As in (i) factor $f$ as $f=h g$ with $h$ proper and $g$ a localizing immersion. Then $\psi(f): f^{\times}=g^{\times} h^{\times} \longrightarrow g^{*} h^{\times}=f^{!}$is the composite

$$
g^{\times} h^{\times} \stackrel{\varepsilon_{1}^{-1} g^{\times} h^{\times}}{\longrightarrow} g^{*} \mathbf{R} g_{*} g^{\times} h^{\times} \stackrel{g^{*} \varepsilon_{2} h^{\times}}{\longrightarrow} g^{*} h^{\times}
$$

where $\varepsilon_{1}: g^{*} \mathbf{R} g_{*} \longrightarrow$ id is the (invertible) counit of the adjunction $g^{*} \dashv \mathbf{R} g_{*}$, while $\varepsilon_{2}: \mathbf{R} g_{*} g^{\times} \longrightarrow$ id is the counit of the adjunction $\mathbf{R} g_{*} \dashv g^{\times}$.

Once again it isn't obvious that the map $\psi(f)$ is independent of the factorization, the reader is referred to [7, Corollary 2.1.4] for the proof. Below we will recall how the trace map was defined in [7] in terms of the $\psi(f)$. The point of this short note is that all of this abstraction is easy to compute.

Assume $f: X \longrightarrow Y$ is a flat morphism in $\mathbb{S}_{\mathbf{e}}$. Let $\pi_{1}, \pi_{2}$ be the two projections $X \times_{Y} X \longrightarrow X$ and let $\delta: X \longrightarrow X \times_{Y} X$ be the diagonal inclusion. We begin with a result of Alonso, Jeremías and Lipman [1, Example 2.4 and Proposition 2.4.2]: they define a morphism $c_{f}$ to be the composite

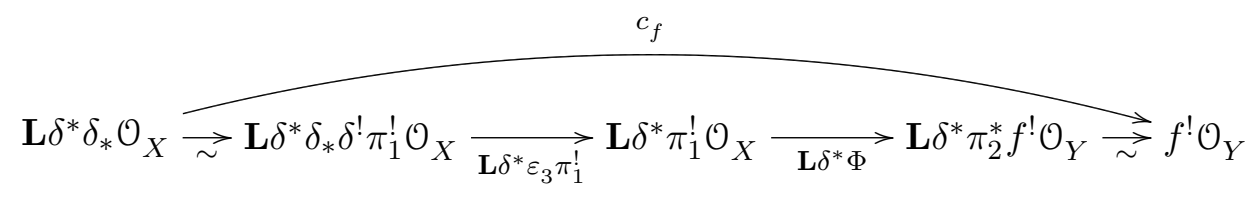

where $\varepsilon_{3}: \delta_{*} \delta^{!}=\delta_{*} \delta^{\times} \longrightarrow$ id is the counit of adjunction $\delta_{*} \dashv \delta^{\times}$, and $\Phi: \pi_{1}^{!} \mathcal{O}_{X}=$ $\pi_{1}^{!} f^{*} \mathcal{O}_{Y} \longrightarrow \pi_{2}^{*} f^{!} \mathcal{O}_{Y}$ is the inverse of the base-change map. If $d$ is any integer then the $t$-structure truncation allows us to extend this to a map

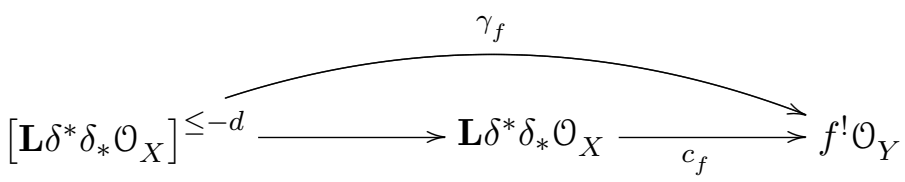

and [1, Proposition 2.4.2] proves $\gamma_{f}$ to be an isomorphism if $f$ is essentially smooth of relative dimension $d$. Note that $H\left[\mathbf{L} \delta^{*} \delta_{*} \mathcal{O}_{X}\right]$ is the Hochschild homology of $\mathcal{O}_{X}$ over $\mathcal{O}_{Y}$, which is a graded commutative sheaf of rings. Since $H H_{1}\left(\mathcal{O}_{X}\right) \cong \Omega_{X / Y}^{1}$ is the sheaf of differential 1-forms the product in the ring $H H_{*}\left(\mathcal{O}_{X}\right)$ gives a natural map $\Omega_{X / Y}^{n}=$ $\wedge^{n} \Omega_{X / Y}^{1} \longrightarrow H H_{n}\left(\mathcal{O}_{X}\right)$, which is an isomorphism for every $n$ if $X$ is essentially smooth over $Y$. If $X$ is essentially smooth over $Y$ of relative dimension $d$ then $\left[\mathbf{L} \delta^{*} \delta_{*} \mathcal{O}_{X}\right]^{\leq-d}$ is just another way of writing $\Sigma^{d} \Omega_{X / Y}^{d}$.

It has been known for a long time that $f^{!} \mathcal{O}_{X / Y}$ is isomorphic to $\Sigma^{d} \Omega_{X / Y}^{d}$; the reader can see Grothendieck [3], or Verdier [12, Theorem 3, p. 397] which relies on Hartshorne [4, III, Proposition 7.2]. What is different about the approach in Alonso, Jeremías and Lipman [1, Example 2.4] is that the map $\gamma_{f}: \Sigma^{d} \Omega_{X / Y}^{d} \longrightarrow f^{!} \mathcal{O}_{X / Y}$ is defined in a global, coordinate-free, functorial way, without making any auxiliary choices. And the local problem becomes to show that this globally defined map is an isomorphism. 
Let $W \subset X$ be the union $W=\cup Z_{i}$ of closed subsets $Z_{i}$ so that the restriction of $f$ to each $Z_{i}$ is proper, let $\mathbf{R} \Gamma_{W}$ be the functor from $\mathbf{D}_{\mathbf{q c}}(X)$ to itself which takes a $K$-injective complex to the largest subcomplex supported on $W$, and let $I: \mathbf{R} \Gamma_{W} \longrightarrow$ id be the canonical inclusion. From [7, Proposition 2.3.2] we learn that $\mathbf{R} \Gamma_{W} \psi(f): \mathbf{R} \Gamma_{W} f^{\times} \longrightarrow$ $\mathbf{R} \Gamma_{W} f^{!}$is an isomorphism, and in [7, Remark 2.3.4] we define the integral $\int_{W}$ to be the composite

$$
\mathbf{R} f_{*} \mathbf{R} \Gamma_{W} f^{!} \underset{\mathbf{R} f_{*} \mathbf{R} \Gamma_{W} \psi(f)^{-1}}{\longrightarrow} \mathbf{R} f_{*} \mathbf{R} \Gamma_{W} f^{\times} \underset{\mathbf{R} f_{*} I f^{\times}}{\int_{W}} \rightarrow \mathbf{R} f_{*} f^{\times} \stackrel{\varepsilon_{4}}{\longrightarrow} \text { id }
$$

where $\varepsilon_{4}$ is the counit of the adjunction $\mathbf{R} f_{*} \dashv f^{\times}$. Putting the two together we have a composite map

$$
\mathbf{R} f_{*} \mathbf{R} \Gamma_{W}\left[\mathbf{L} \delta^{*} \delta_{*} \mathcal{O}_{X}\right]^{\leq-d} \longrightarrow \mathbf{R} f_{*} \mathbf{R} \Gamma_{W} \gamma_{f} \longrightarrow \mathbf{R} f_{*} \mathbf{R} \Gamma_{W} f^{!} \mathcal{O}_{Y} \stackrel{\int_{W}}{\longrightarrow} \mathcal{O}_{Y}
$$

and the point of this article is that we will compute it in the affine, essentially smooth case. Since everything commutes with étale base change and all smooth morphisms are étale locally projections $\mathbb{A}_{Y}^{d} \longrightarrow Y$, the key is to compute this case.

Once again there are integrals defined in the literature, starting with Hartshorne [4, III.9 and 10], but see also Lipman [8], Hübl and Kunz [5, 6] and Sastry [11, Section 3.1]. What is rare is to have a definition that is global, coordinate-free, functorial and computable. Note that in the preceding page we have given complete and self-contained definitions for all the maps we will consider, and they are clearly globally defined by functorial formulas. In the rest of the article we will show them easy to compute.

The one caveat is that we have not said much about the morphism $\psi(f): f^{\times} \longrightarrow$ $f^{!}$beyond giving the definition; this is somehow the really new ingredient, a natural transformation defined only very recently in [7].

Sancho de Salas [10] presents a nice characterization of residues, in terms of some properties they satisfy. In our special case the direct computation is so easy, it seemed unnecessary to go through Sancho de Salas' formalism.

Acknowlegements. The author would like to thank Joe Lipman and an anonymous referee for suggestions that greatly improved the presentation.

\section{THE MAP $\gamma_{f}$ IS AN ISOMORPHISM}

In $\left[1\right.$, Proposition 2.4.2] there is a proof that the map $\gamma_{f}$ is an isomorphism for $f$ essentially smooth of relative dimension $d$; in this section we present a somewhat different argument. The proof in [1, Proposition 2.4.2] appeals to [12, Theorem 3, p. 397] whereas the treatment here is based on the results of [7]. 
Remark 1.1. This makes the approach here different in spirit from the one of $[1$, Proposition 2.4.2]. Verdier's [12, Theorem 3, p. 397], which is key to the proof of [1, Proposition 2.4.2], relies on Hartshorne's isomorphism [4, Proposition III.7.2, pp. 179-180], an isomorphism constructed locally using coordinates and then shown to glue to a global map. The results we appeal to in [7] are all formal, about some globally defined diagrams commuting or some composites being isomorphisms. Unlike the argument in [1, Proposition 2.4.2] the one here contains the entire local computation. There is no gluing anywhere - we have globally defined, abstract nonsense maps, and using formal properties about them we show locally that some composite is an isomorphism.

Remark 1.2. Back to proving that $\gamma_{f}$ is an isomorphism. The question is local in $X$ and $Y$, hence we may assume them both affine. Let $X=\operatorname{Spec}(S), Y=\operatorname{Spec}(R)$, and assume $f$ is induced by an essentially smooth ring homomorphism $\sigma: R \longrightarrow S$, of relative dimension $d$. Shrinking $X$ if necessary, we may assume that the map $f: X \longrightarrow Y$ factors as $X \stackrel{g}{\longrightarrow} \mathbb{A}_{Y}^{d} \stackrel{h}{\longrightarrow} Y$ with $g$ essentially étale and $h$ the projection from the affine space $\mathbb{A}_{Y}^{d}$ to $Y$. The composite defining $\gamma_{f}$ consists of maps

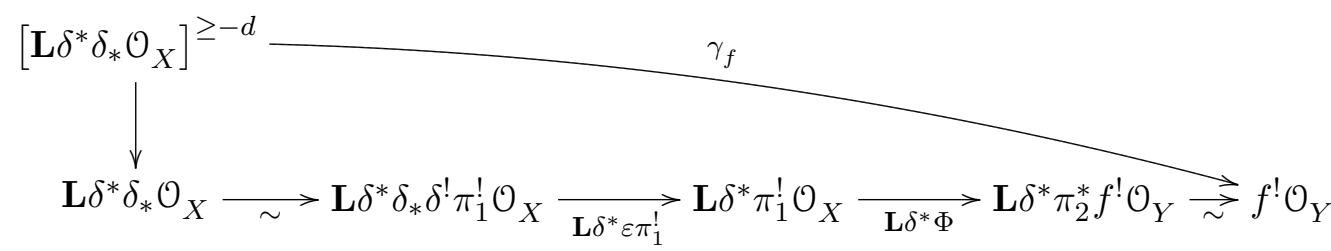

all of which commute with étale base change; this means that $\gamma_{f}$ is naturally isomorphic to $g^{*} \gamma_{h}$, and it suffices to prove that $\gamma_{h}$ is an isomorphism. In other words we are reduced to considering the case where $Y=\operatorname{Spec}(R)$ and $X=\operatorname{Spec}\left(R\left[x_{1}, x_{2}, \ldots, x_{d}\right]\right)$.

Remark 1.3. First some notation: for a scheme $X$ let $\mathbf{D}_{\mathbf{q c}}(X)$ be the derived category of complexes of sheaves of $\mathcal{O}_{X}$-modules with quasicoherent cohomology. If $X=\operatorname{Spec}(S)$ is affine then there is an equivalence of categories $\mathbf{D}_{\mathbf{q c}}(X) \cong \mathbf{D}(S)$, where $\mathbf{D}(S)$ is the derived category of $S$-modules; see [2, Lemma 5.4]. We will freely confuse $\mathbf{D}_{\mathbf{q c}}(X)$ with the equivalent $\mathbf{D}(S)$. Thus we will write $S$ for the corresponding object in $\mathbf{D}_{\mathbf{q c}}(\operatorname{Spec}(S))$, that is for the structure sheaf $S^{\sim}=\mathcal{O}_{X}$. And if $\sigma: R \longrightarrow S$ is a ring homomorphism inducing a map $f=\operatorname{Spec}(\sigma): \operatorname{Spec}(S) \longrightarrow \operatorname{Spec}(R)$, we will write $f^{\times} R$ and $f^{!} R$ for what we should really denote $f^{\times} \mathcal{O}_{\operatorname{Spec}(R)}$ and $f^{!} \mathcal{O}_{\operatorname{Spec}(R)}$.

In this notation, [7, Theorem 4.2.4] computes for us the map $\gamma_{f}$ in the affine, flat situation. For our flat ring homomorphism $\sigma: R \longrightarrow S$ the map $\gamma_{f}$ comes down to the composite

$$
\left[S \otimes_{S^{\mathrm{e}}}^{\mathbf{L}} S\right]^{\leq-d} \longrightarrow S \otimes_{S^{\mathrm{e}}}^{\mathbf{L}} S \stackrel{c_{f}}{\stackrel{\mathrm{id} \otimes \mu}{\longrightarrow} S \otimes_{S^{\mathrm{e}}}^{\mathbf{L}} \operatorname{Hom}_{R}(S, S) \longrightarrow S \otimes_{S^{\mathrm{e}}}^{\mathbf{L}}} \mathbf{R H o m}_{R}(S, S)
$$


where the general map $c_{f}$ specializes to the composite shown, and where $\mu$ is the inclusion $S \longrightarrow \operatorname{Hom}_{R}(S, S)$ taking $s \in S$ to the homomorphism $s: S \longrightarrow S$ which multiplies by $s$. In this section we will prove directly that $\gamma_{f}$ is an isomorphism when $S$ is the polynomial ring over $R$. Observe that in this special case $S$ is projective as an $R$-module, and hence the natural map $\operatorname{Hom}_{R}(S, S) \longrightarrow \mathbf{R H o m}_{R}(S, S)$ is an isomorphism. The composite we need to compute therefore shortens to

$$
\left[S \otimes_{S^{\mathrm{e}}}^{\mathbf{L}} S\right]^{\leq-d} \longrightarrow S \otimes_{S^{\mathrm{e}}}^{\mathbf{L}} S \longrightarrow \text { id } \otimes \mu \quad \longrightarrow \otimes_{S^{\mathrm{e}}}^{\mathbf{L}} \operatorname{Hom}_{R}(S, S) .
$$

We begin with two lemmas. For the application in this section we only need half of these lemmas, in the notation of Lemma 1.4 we only need to know the kernel of the map $r_{x}-\ell_{x}$. But we will need the cokernel of $r_{x}-\ell_{x}$ in Section 2.

Lemma 1.4. Let $S \longrightarrow T \longrightarrow T[x]$ be ring homomorphisms, with $T[x]$ being the polynomial ring in one variable over $T$. There is a short exact sequence

$$
0 \longrightarrow \operatorname{Hom}_{S[x]}(T[x], T[x]) \stackrel{i}{\longrightarrow} \operatorname{Hom}_{S}(T[x], T[x]) \stackrel{r_{x}-\ell_{x}}{\longrightarrow} \operatorname{Hom}_{S}(T[x], T[x]) \longrightarrow 0
$$

where $i$ is the natural inclusion, and $r_{x}-\ell_{x}$ takes an $S$-linear map $\rho: T[x] \longrightarrow T[x]$ to $\rho x-x \rho$, the difference between right multiplication by $x$ and left multiplication by $x$.

Proof. We have to study the kernel and cokernel of the map $\left(r_{x}-\ell_{x}\right)$. We remind the reader: the way $r_{x}-\ell_{x}$ acts on $\operatorname{Hom}_{S}(T[x], T[x])$ is that it takes a linear map $\rho: T[x] \longrightarrow T[x]$ to the map $\left(r_{x}-\ell_{x}\right) \rho$, where

$$
\left[\left(r_{x}-\ell_{x}\right) \rho\right](P)=\rho(x P)-x \rho(P) .
$$

Thus $\rho$ belongs to the kernel if and only if $\rho(x P)=x \rho(P)$, which is if and only if $\rho$ is $S[x]$-linear. This identified the kernel; next we have to prove that $\left(r_{x}-\ell_{x}\right)$ : $\operatorname{Hom}_{S}(T[x], T[x]) \longrightarrow \operatorname{Hom}_{S}(T[x], T[x])$ is surjective. Let $\varphi: T[x] \longrightarrow T[x]$ be an element of $\operatorname{Hom}_{S}(T[x], T[x])$, we must exhibit it as $\left(r_{x}-\ell_{x}\right) \rho$. We define $\rho: T[x] \longrightarrow T[x]$ inductively by the rule

(i) $\rho(t)=0$ for all $t \in T$.

(ii) Assume $\rho\left(t x^{i}\right)$ has been defined for all $t \in T$ and all $i \leq n$. Then $\rho\left(t x^{n+1}\right)=$ $\varphi\left(t x^{n}\right)+x \rho\left(t x^{n}\right)$.

The $S$-linearity of $\varphi$ and the inductive formula guarantee that $\rho$ will be $S$-linear. The inductive formula also tells us that $\varphi\left(t x^{n}\right)=\rho\left(x \cdot t x^{n}\right)-x \rho\left(t x^{n}\right)=\left[\left(r_{x}-\ell_{x}\right) \rho\right]\left(t x^{n}\right)$. But then $\varphi$ and $\left(r_{x}-\ell_{x}\right) \rho$ are linear maps that agree on all elements of the form $t x^{n} \in T[x]$, which span $T[x]$.

Lemma 1.5. Suppose we are given homomorphisms of rings $R \longrightarrow S \longrightarrow T \longrightarrow T[x]$, and let $T[x]^{\mathrm{e}}=T[x] \otimes_{R} T[x]$. Let $\mu_{S}: T[x] \longrightarrow \operatorname{Hom}_{S}(T[x], T[x])$ be the map that takes $P \in T[x]$ to the map $P: T[x] \longrightarrow T[x]$ which multiplies by $P$. Then the tensor product over $T[x]^{\mathrm{e}}$ of $\mu_{S}$ with the complex

$$
\cdots \longrightarrow 0 \longrightarrow T[x]^{\mathrm{e}} \stackrel{x \otimes 1-1 \otimes x}{\longrightarrow} T[x]^{\mathrm{e}} \longrightarrow 0 \longrightarrow \cdots
$$


is isomorphic in $\mathbf{D}\left(T[x]^{\mathrm{e}}\right)$ to a map

$$
T[x] \oplus \Sigma T[x] \longrightarrow \Sigma \operatorname{Hom}_{S[x]}(T[x], T[x])
$$

so that the composite

$$
\Sigma T[x] \stackrel{\text { inclusion }}{\longrightarrow} T[x] \oplus \Sigma T[x] \longrightarrow \Sigma \operatorname{Hom}_{S[x]}(T[x], T[x])
$$

is just the suspension of the map $\mu_{S[x]}: T[x] \longrightarrow \operatorname{Hom}_{S[x]}(T[x], T[x])$.

Proof. If we tensor the complex $T[x]^{\mathrm{e}} \stackrel{x \otimes 1-1 \otimes x}{\longrightarrow} T[x]^{\mathrm{e}}$ with the map $\mu_{S}$ we obtain a chain map

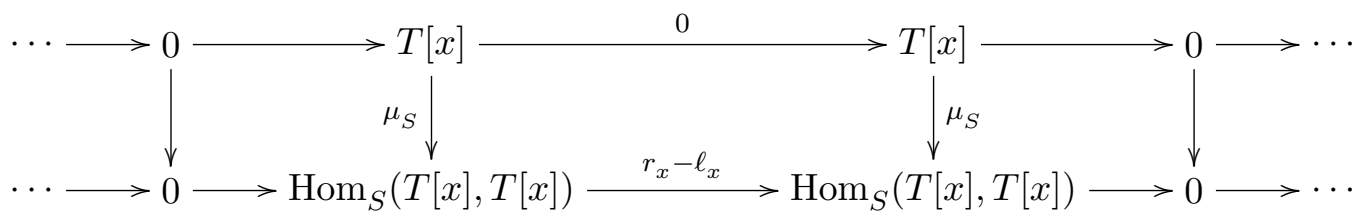

the Lemma now follows from Lemma 1.4 and the observation that the map $\mu_{S}: T[x] \longrightarrow$ $\operatorname{Hom}_{S}(T[x], T[x])$ factors as

$$
T[x] \stackrel{\mu_{S[x]}}{\longrightarrow} \operatorname{Hom}_{S[x]}(T[x], T[x]) \stackrel{i}{\longrightarrow} \operatorname{Hom}_{S}(T[x], T[x])
$$

where $i$ is the natural inclusion, as in Lemma 1.4.

Proposition 1.6. Let $f: X \longrightarrow Y$ be an essentially smooth morphism of relative dimension d. Then the map $\gamma_{f}$ of (0.0.1) is an isomorphism.

Proof. By Remark 1.2 it suffices to consider the case $Y=\operatorname{Spec}(R), X=\operatorname{Spec}\left(S_{d}\right)$ with $S_{d}=R\left[x_{1}, x_{2}, \ldots, x_{d}\right]$ the polynomial ring in $d$ variables. We want to study the map $\gamma_{f}$, and by Remark 1.3 it is the composite

$$
\left[S_{d} \otimes S_{d}^{\mathbf{e}} S_{d}\right]^{\leq-d} \longrightarrow S_{d} \otimes_{S_{d}^{\mathrm{e}}}^{\mathbf{L}} S_{d} \longrightarrow S_{d} \otimes_{S_{d}^{\mathrm{e}}}^{\mathbf{L}} \operatorname{Hom}_{R}\left(S_{d}, S_{d}\right)
$$

We will prove the Proposition by induction on $n$ with $0 \leq n \leq d$. More precisely:

1.6.1. Consider for each $n$ the ring $S_{n}=k\left[x_{1}, x_{2}, \ldots, x_{n}\right]$ and the Koszul complex

$$
K_{n} \cong \bigotimes_{i=1}^{n}\left(S_{d}^{\mathrm{e}} \stackrel{x_{i} \otimes 1-1 \otimes x_{i}}{\longrightarrow} S_{d}^{\mathrm{e}}\right)
$$

For each $n$ there is the composite

$$
\left[K_{n} \otimes_{S_{d}^{\mathrm{e}}} S_{d}\right]^{\leq-n} \stackrel{\text { inclusion }}{\longrightarrow} K_{n} \otimes_{S_{d}^{\mathrm{e}}} S_{d} \stackrel{\mathrm{id} \otimes \mu}{\longrightarrow} K_{n} \otimes_{S_{d}^{\mathrm{e}}} \operatorname{Hom}_{R}\left(S_{d}, S_{d}\right)
$$

We will prove

(i) The composite is isomorphic in the derived category $\mathbf{D}\left(S_{d}^{\mathrm{e}}\right)$ to $\Sigma^{n}$ applied to the inclusion $\mu_{S_{n}}: S_{d} \longrightarrow \operatorname{Hom}_{S_{n}}\left(S_{d}, S_{d}\right)$

(ii) The natural map $\left[K_{n} \otimes_{S_{d}^{\mathrm{e}}} S_{d}\right]^{\leq-n} \longrightarrow K_{n} \otimes_{S_{d}^{\mathrm{e}}} S_{d}$ is split injective. 
The statement (i) for $n=d$ gives the Proposition, as soon as we observe that $K_{d}$ is a projective resolution for $S_{d}$ over $S_{d}^{\mathrm{e}}$.

Since both (i) and (ii) are trivially true when $n=0$ it remains to prove the induction step. Suppose we know (i) and (ii) for $n$. Because (ii) is true for $n$ we have that $K_{n} \otimes_{S_{d}^{\mathrm{e}}} S_{d} \cong\left[K_{n} \otimes_{S_{d}^{\mathrm{e}}} S_{d}\right]^{\leq-n} \oplus\left[K_{n} \otimes_{S_{d}^{\mathrm{e}}} S_{d}\right]^{\geq-n+1}$, and we put this together with (ii) to obtain a commutative diagram

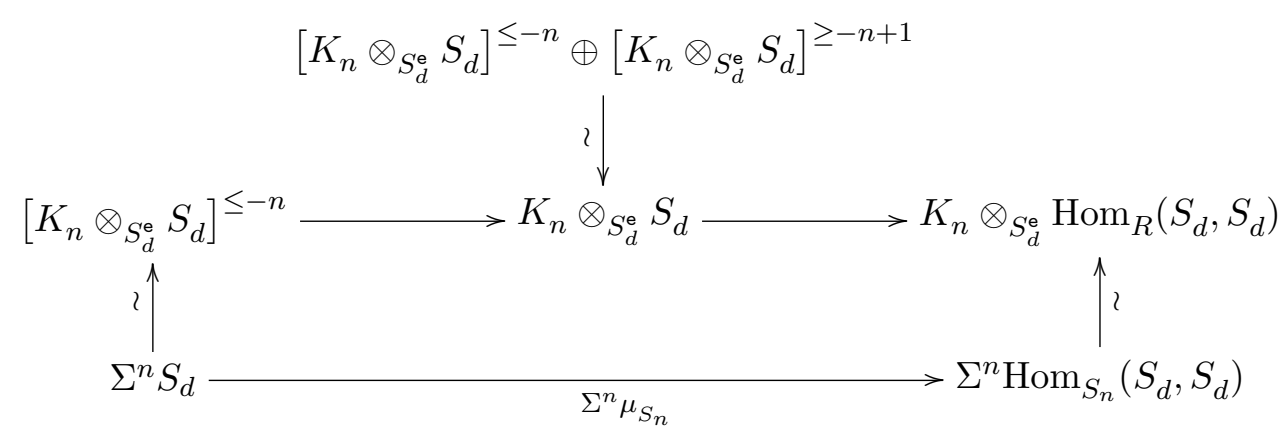

where the vertical maps are isomorphisms. The inductive step follows by tensoring this commutative diagram with

$$
S_{d}^{\mathrm{e}} \stackrel{x_{n+1} \otimes 1-1 \otimes x_{n+1}}{\longrightarrow} S_{d}^{\mathrm{e}}
$$

and applying Lemma 1.5 to the bottom row with $S=S_{n}$, with $T$ the polynomial ring $S_{n}\left[x_{n+2}, \ldots, x_{d}\right]$, and where $T\left[x_{n+1}\right]=S_{d}$.

Remark 1.7. (1.6.1)(i) for $n=d$ gives an isomorphism $\left[S_{d} \otimes_{S_{d}^{\mathrm{e}}}^{\mathbf{L}} S_{d}\right]{ }^{\leq-d} \cong \Sigma^{d} S_{d}$. Of course $\left[S_{d} \otimes_{S_{d}^{\mathrm{e}}}^{\mathbf{L}} S_{d}\right]^{\leq-d}$ is naturally isomorphic to the sheaf of differentials $\Omega_{S_{d}}^{d} / R$, but this line bundle is trivial over Spec of the polynomial ring $S_{d}$. We chose not to complicate the notation by writing differential forms everywhere.

Remark 1.8. The case $d=1$ of (1.6.1) tells us that the chain map

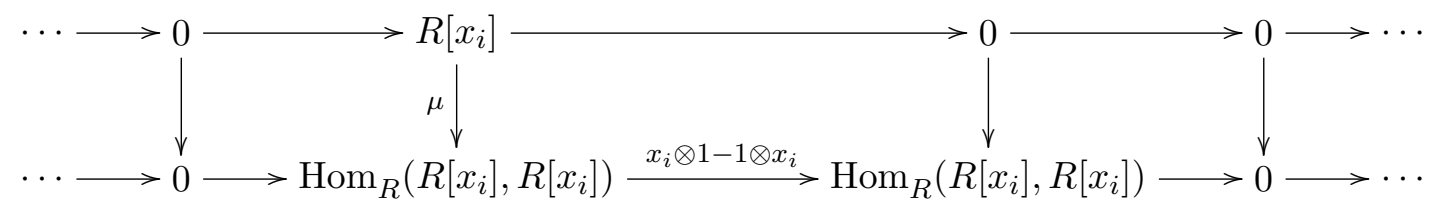

is a quasi-isomorphism. Tensoring over $R$, as $i$ ranges in the interval $1 \leq i \leq d$, we deduce a quasi-isomorphism

$$
\Sigma^{d} S_{d} \stackrel{\Theta}{\longrightarrow} \bigotimes_{i=1}^{d}\left[\operatorname{Hom}_{R}\left(R\left[x_{i}\right], R\left[x_{i}\right]\right) \stackrel{x_{i} \otimes 1-1 \otimes x_{i}}{\longrightarrow} \operatorname{Hom}_{R}\left(R\left[x_{i}\right], R\left[x_{i}\right]\right)\right]
$$

and the quasi-isomorphism of Proposition 1.6 is closely related. We have a map

$$
\bigotimes_{i=1}^{d} \operatorname{Hom}_{R}\left(R\left[x_{i}\right], R\left[x_{i}\right]\right) \stackrel{\Psi}{\longrightarrow} \operatorname{Hom}_{R}\left(R\left[x_{1}, x_{2}, \ldots, x_{d}\right], R\left[x_{1}, x_{2}, \ldots, x_{d}\right]\right)
$$


which we may tensor with the complex $K_{d}$ of (1.6.1). The reader can check that the composite $\left(K_{d} \otimes \Psi\right) \circ \Theta$ is the quasi-isomorphism of Proposition 1.6. By the above $\Theta$ is also a quasi-isomorphism; hence so is $K_{d} \otimes \Psi$.

\section{Residues}

Lemma 2.1. Let $R$ be a ring. The chain map

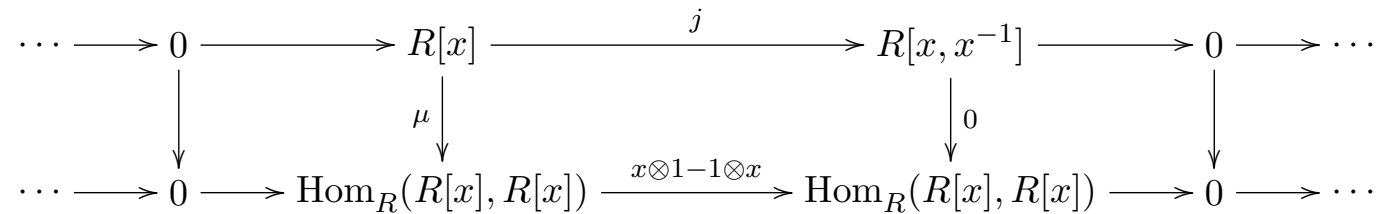

is homotopic to the chain map

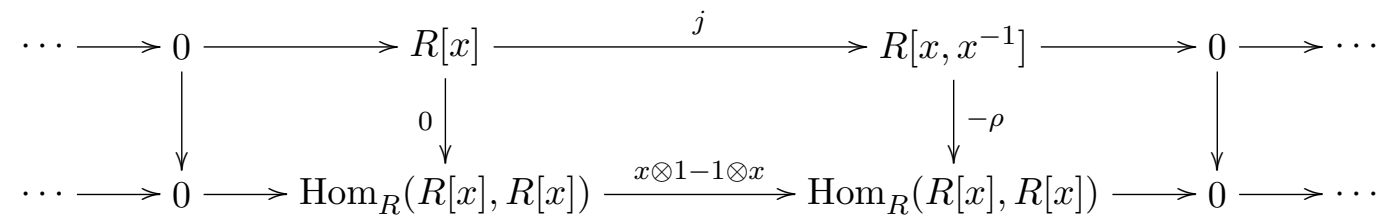

where for $P \in R\left[x, x^{-1}\right]$ the element $\rho(P) \in \operatorname{Hom}_{R}(R[x], R[x])$ is the function $\rho(P)$ : $R[x] \longrightarrow R[x]$ taking a polynomial $Q$ to the residue of $P Q$ at 0 , in other words the coefficient of $x^{-1}$ in the Laurent polynomial $P Q$.

Proof. We need to specify a homotopy $\Theta: R\left[x, x^{-1}\right] \longrightarrow \operatorname{Hom}_{R}(R[x], R[x])$. It is given by the following rule: if $P \in R\left[x, x^{-1}\right]$ then $\Theta(P): R[x] \longrightarrow R[x]$ takes $Q \in R[x]$ to

$$
[\Theta(P)](Q)=[P Q]^{\geq 0} .
$$

The truncation $L^{\geq 0}$ for the Laurent polynomial $L=\sum_{n=-\infty}^{\infty} r_{n} x^{n}$ is given by $L^{\geq 0}=$ $\sum_{n=0}^{\infty} r_{n} x^{n}$. We truncate all the terms with negative exponents.

If $P \in R[x] \subset R\left[x, x^{-1}\right]$ then $P Q$ is a polynomial and $[P Q]^{\geq 0}=P Q$; therefore $\Theta j=\mu$. To compute $(x \otimes 1-1 \otimes x) \Theta$ observe that

$$
\begin{aligned}
{[(x \otimes 1-1 \otimes x) \Theta(P)](Q) } & =\Theta(P)(x Q)-x \Theta(P)(Q) \\
& =[P x Q]^{\geq 0}-x[P Q]^{\geq 0}
\end{aligned}
$$

which is the coefficient of $x^{-1}$ in the Laurent polynomial $P Q$.

Lemma 2.2. Let $K_{d}$ be the Koszul complex of (1.6.1), and let $\gamma_{f}$ be the map of (0.0.1) as computed in (1.6.1). Let $R\left[x_{i}\right] \stackrel{j_{i}}{\longrightarrow} R\left[x_{i}, x_{i}^{-1}\right]$ be the complex with $R\left[x_{i}\right]$ in degree -1 , with $R\left[x_{i}, x_{i}^{-1}\right]$ in degree 0 , and zeros in all other degrees. Consider the composite

$$
\bigotimes_{i=1}^{d}\left(R\left[x_{i}\right] \stackrel{j_{i}}{\longrightarrow} R\left[x_{i}, x_{i}^{-1}\right]\right) \stackrel{\widetilde{I}}{\longrightarrow} \Sigma^{d} S_{d} \stackrel{\gamma_{f}}{\longrightarrow} K_{d} \otimes_{S_{d}^{e}} \operatorname{Hom}_{R}\left(S_{d}, S_{d}\right)
$$


where $\widetilde{I}$ is the map which is the identity in degree - $d$ and zero in all other degrees. Then the composite above is homotopic to the map

$$
\bigotimes_{i=1}^{d}\left(R\left[x_{i}\right] \stackrel{j_{i}}{\longrightarrow} R\left[x_{i}, x_{i}^{-1}\right]\right) \stackrel{(-1)^{d} \alpha}{\longrightarrow} \operatorname{Hom}_{R}\left(S_{d}, R\right) \stackrel{\psi(f)}{\longrightarrow} K_{d} \otimes_{S_{d}^{\mathrm{e}}} \operatorname{Hom}_{R}\left(S_{d}, S_{d}\right)
$$

where $\psi(f)$ is the map of [7, Proposition 3.2.9] in the case where $M=R$ is the trivial $R$-module, and $\alpha$ is given in degree 0 by the homomorphism $\alpha^{0}: \otimes_{i=1}^{d} R\left[x_{i}, x_{i}^{-1}\right] \longrightarrow$ $\operatorname{Hom}_{R}\left(S_{d}, R\right)$ which takes $P \in \otimes_{i=1}^{d} R\left[x_{i}, x_{i}^{-1}\right]$ to the map taking $Q \in S_{d}$ to the residue of $P Q$ at 0 , that is the coefficient of $x_{1}^{-1} x_{2}^{-1} \cdots x_{d}^{-1}$.

Remark 2.3. To see that $\alpha$ is a well-defined map of complexes we need to show that $\alpha^{0}: \otimes_{i=1}^{d} R\left[x_{i}, x_{i}^{-1}\right] \longrightarrow \operatorname{Hom}_{R}\left(S_{d}, R\right)$ vanishes on the image of the differential of the complex $C=\bigotimes_{i=1}^{d}\left(R\left[x_{i}\right] \stackrel{j_{i}}{\longrightarrow} R\left[x_{i}, x_{i}^{-1}\right]\right)$. The point is that the residue of $P Q$ will vanish if there is some $x_{i}$ so that $P$ involves only nonnegative powers of $x_{i}$.

Proof. By Lemma 2.1 we have that the tensor product over $R$, as $i$ ranges over $1 \leq i \leq d$, of the chain maps

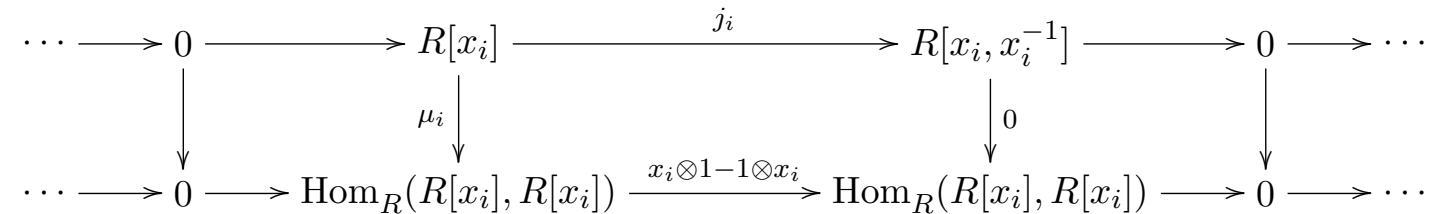

is homotopic to the tensor product over $R$, as $i$ ranges over $1 \leq i \leq d$, of the chain maps

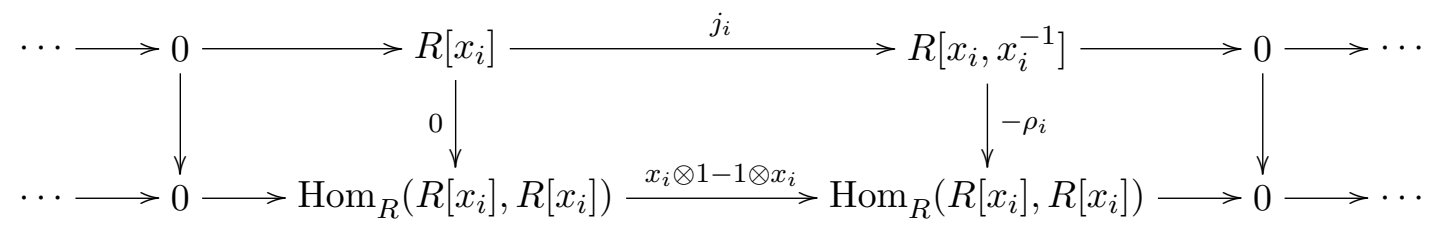

The Lemma follows immediately if we compose these homotopic maps with the quasiisomorphism $K_{d} \otimes \Psi$ of Remark 1.8.

Remark 2.4. Let $W \subset \operatorname{Spec}\left(S_{d}\right)$ be the closed set given by the ideal $\left(x_{1}, x_{2}, \ldots, x_{d}\right)$. Then the map $\tilde{I}: C=\bigotimes_{i=1}^{d}\left(R\left[x_{i}\right] \stackrel{j_{i}}{\longrightarrow} R\left[x_{i}, x_{i}^{-1}\right]\right) \longrightarrow \Sigma^{d} S_{d}$ of Lemma 2.2 can be completed to a triangle

$$
C \stackrel{\widetilde{I}}{\longrightarrow} \Sigma^{d} S_{d} \longrightarrow Y \longrightarrow \Sigma C
$$

where $C$ is supported on $W$ while $Y$ is a finite complex of modules whose direct summands have at least one $x_{i}$ acting invertibly, and hence $\operatorname{RHom}(J, Y)=0$ for all $J$ supported on $W$. Therefore the map $\widetilde{I}: C \longrightarrow \Sigma^{d} S_{d}$ identifies canonically with $I: \mathbf{R} \Gamma_{W} \Sigma^{d} S_{d} \longrightarrow$ 
$\Sigma^{d} S_{d}$. That is Lemma 2.2 can be viewed as giving a commutative square in the derived category

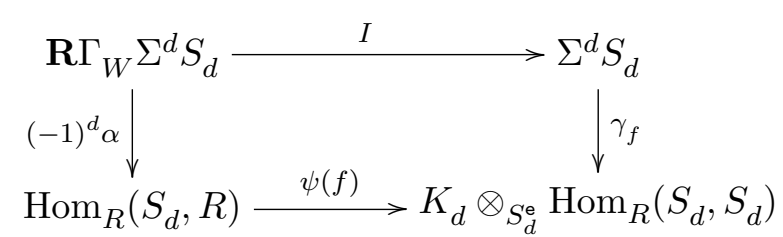

Now $I: \mathbf{R} \Gamma_{W} \longrightarrow$ id is a natural transformation so the following diagram commutes

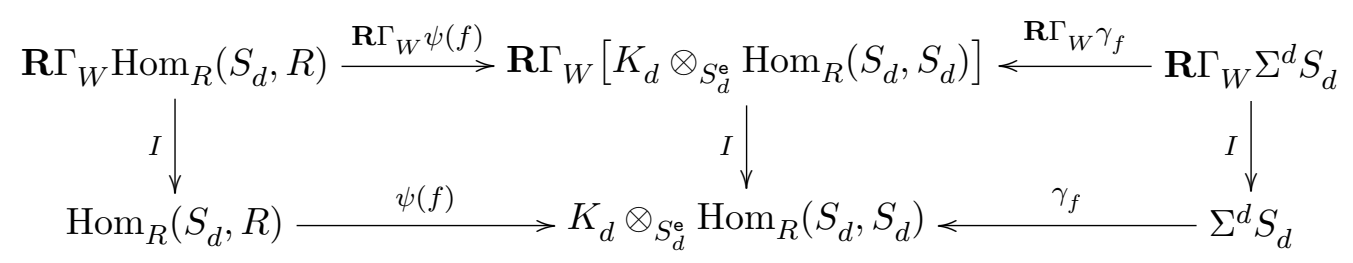

and the map $(-1)^{d} \alpha: \mathbf{R} \Gamma_{W} \Sigma^{d} S_{d} \longrightarrow \operatorname{Hom}_{R}\left(S_{d}, R\right)$ of Lemma 2.2 is a morphism from an object supported on $W$ to $\operatorname{Hom}_{R}\left(S_{d}, R\right)$ and therefore factors uniquely as

$$
\mathbf{R} \Gamma_{W} \Sigma^{d} S_{d} \stackrel{\varphi}{\longrightarrow} \mathbf{R} \Gamma_{W} \operatorname{Hom}_{R}\left(S_{d}, R\right) \stackrel{I}{\longrightarrow} \operatorname{Hom}_{R}\left(S_{d}, R\right) .
$$

Consider now the following diagram

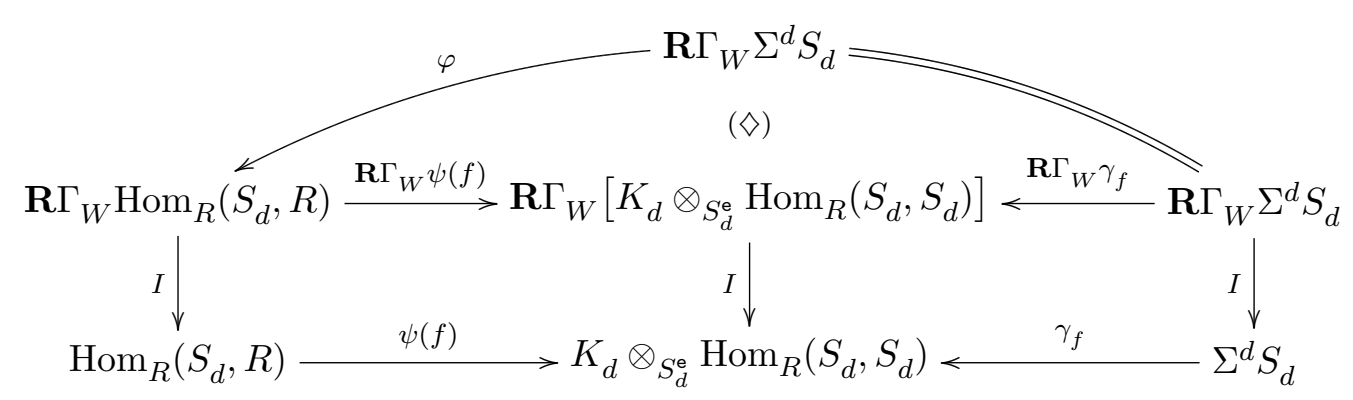

Lemma 2.2 coupled with the definition of $\varphi$ tell us that the perimeter commutes. But then the two composites in the triangle $(\diamond)$ compose to the same map when followed by $I: \mathbf{R} \Gamma_{W}\left[K_{d} \otimes_{S_{d}^{\mathrm{e}}} \operatorname{Hom}_{R}\left(S_{d}, S_{d}\right)\right] \longrightarrow K_{d} \otimes_{S_{d}^{\mathrm{e}}} \operatorname{Hom}_{R}\left(S_{d}, S_{d}\right)$, and must agree. Thus the triangle

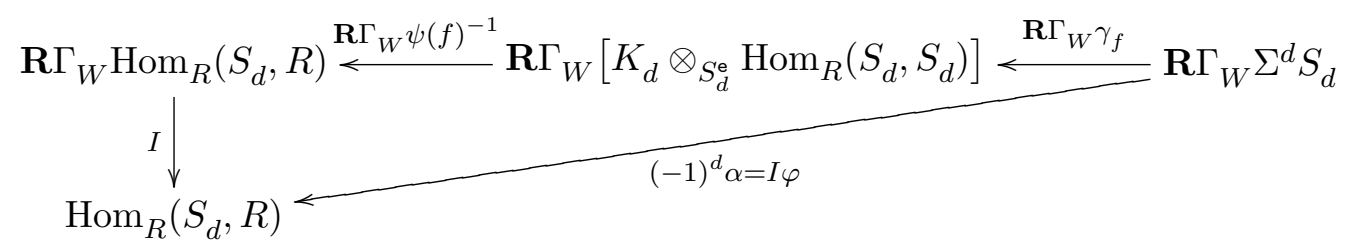

commutes, and if we apply $\mathbf{R} f_{*}$ to this commutative triangle and follow with the counit of adjunction $\varepsilon: \mathbf{R} f_{*} \operatorname{Hom}_{R}\left(S_{d}, R\right) \longrightarrow R$ then we obtain the composite (0.0.2) of the Introduction. The counit of adjunction $\varepsilon: \mathbf{R} f_{*} f^{\times} R=\operatorname{Hom}_{R}\left(S_{d}, R\right) \longrightarrow R$ is standard, it is the map that evaluates a $g: S_{d} \longrightarrow R$ at $1 \in S_{d}$; see also [7, Lemma 3.1] for a 
global (non-affine) version. Therefore the composite in (0.0.2) of the Introduction takes a Laurent polynomial $P \in \otimes_{i=1}^{d} R\left[x_{i}, x_{i}^{-1}\right]$ to $(-1)^{d}[\alpha(P)](1)$, which is $(-1)^{d}$ times the coefficient of $x_{1}^{-1} x_{2}^{-1} \cdots x_{d}^{-1}$ in the Laurent polynomial $P 1=P$.

We summarize the result of the computation in Remark 2.4:

Corollary 2.5. In the case where $f: X \longrightarrow Y$ is induced by the inclusion $R \longrightarrow$ $S=R\left[x_{1}, x_{2}, \ldots, x_{d}\right]$ and where $W \subset \operatorname{Spec}(S)$ is the closed subset corresponding to the ideal $\left(x_{1}, x_{2}, \ldots, x_{d}\right) \subset S$, the composite of (0.0.2) comes down to $(-1)^{d}$ times the map $\otimes_{i=1}^{d} R\left[x_{i}, x_{i}^{-1}\right] \longrightarrow R$ taking the residue at 0 of a Laurent polynomial $P \in \otimes_{i=1}^{d} R\left[x_{i}, x_{i}^{-1}\right]$.

We leave it to the reader to formulate the consequences that can be obtained by factoring maps as $X \stackrel{g}{\longrightarrow} \mathbb{A}_{Y}^{d} \stackrel{h}{\longrightarrow} Y$ with $g$ essentially étale.

\section{REFERENCES}

1. Leovigildo Alonso Tarrío, Ana Jeremías López, and Joseph Lipman, Bivariance, Grothendieck duality and Hochschild homology II: the fundamental class of a flat scheme-map, (arXiv:1202.4367).

2. Marcel Bökstedt and Amnon Neeman, Homotopy limits in triangulated categories, Compositio Math. 86 (1993), 209-234.

3. Alexandre Grothendieck, Théorèmes de dualité pour les faisceaux algébriques cohérents, Séminaire Bourbaki (May, 1957), no. 149.

4. Robin Hartshorne, Residues and duality, Lecture Notes in Mathematics, vol. 20, Springer-Verlag, 1966.

5. Reinhold Hübl and Ernst Kunz, Integration of differential forms on schemes, J. Reine Angew. Math. 410 (1990), 53-83.

6. __ Regular differential forms and duality for projective morphisms, J. Reine Angew. Math. 410 (1990), 84-108.

7. Srikanth B. Iyengar, Joseph Lipman, and Amnon Neeman, Relation between two twisted inverse image pseudofunctors in duality theory, (http://arxiv.org/abs/1307.7092).

8. Joseph Lipman, Dualizing sheaves, differentials and residues on algebraic varieties, Astérisque (1984), no. $117, \mathrm{ii}+138$.

9. Amnon Neeman, An improvement on the base-change theorem and the functor $f^{!}$, preprint.

10. Fernando Sancho de Salas, Residue: a geometric construction, Canad. Math. Bull. 45 (2002), no. 2, 284-293.

11. Pramathanath Sastry, Base change and Grothendieck duality for Cohen-Macaulay maps, Compos. Math. 140 (2004), no. 3, 729-777.

12. Jean-Louis Verdier, Base change for twisted inverse images of coherent sheaves, vol. Collection: Algebraic Geometry, Tata Inst. Fund. Res., 1968, pp. 393-408.

Centre for Mathematics and its Applications, Mathematical Sciences Institute, John Dedman Bullding, The Australian National University, Canberra, ACT 0200, AUstralia E-mail address: Amnon. Neeman@anu.edu.au 